\title{
A shortest path method on a surface in space
}

\author{
Ahmad Tayyar \\ Jerash Private University, Jordan, ahmad.tayyar@jpu.edu.jo
}

\begin{abstract}
The objective of this work is to determine the shortest path passing through two points of a surface in the space $z=f(x$, $y)$. The presented work firstly highlights the limitations of employing the conventional method which relies on solving the system of second-order differential equations (Euler-Lagrange) in finding effective solutions, then introduce a random numerical method to approach this problem [2].

The method of Euler Lagrange requires the search for initial velocity for differential equations of the second order. This leads to a solution connecting two fixed points of the plan. Here, I modify the initial velocity at one point to find the solutions passing through the other point. Such a solution is called the geodesic path, which passes through these two points and is considered the shortest path between the two points. However, this is not always true (i.e. the case of a sphere) $[1,2]$.

At the beginning of this work, I present a method for the search of the initial velocity of the Euler-Lagrang system. I apply it on real examples in order to illustrate the limits of the efficiency of this method, then I introduce a numerical method based on random changes to determine the shortest path.
\end{abstract}

Key words: Geodesic, Metric, shortest path, Euler Lagrange system.

\section{INTRODUCTION}

This paper presents a numerical method to solve the issue of finding the shortest path between two points of the space, using the generation of random variables.

When the surface is uniform, this issue may be solved by solving differential system of the second level, with boundary conditions; we give an initial velocity at the beginning point to get a solution representing geodesic curve $[1,2]$. We change the value of the initial velocity, and keep repeating, until getting geodesic curve passing through the second point. However, this method is not always applicable and is inefficient when the surface's metric is so sensitive to the change of the initial velocity. Also, for the slowly changing metrics, the search for the suitable velocity is so long [3].

Moreover, the geodesic line that passes through two points is not necessarily the shortest path passing through them (i.e. the case of a sphere). In addition, I was not able to find a reliable method for this issue when the surface contains obstacles, except the case of the Euclidean metric with obstacles of the polygonal shaped obstacles, where the processing occurs by combinatorial approaches.
The numerical method introduced in this paper is based on random changes. I adopt a discrete curve on the computer's screen that represents a curve on a surface in the space and passes through two stationary points. I then change the positions of the mentioned points by performing random transitions in all directions, and in all dimensions. I adopt these transitions if they reduce the total length of the curve. I repeat this procedure on all points that connect the two stationary points, until gaining the maximum reduction of the curve's length; this means that any further change on the curve's points will not be advantageous.

This research has various applications. For instance, it can be implemented in photo processing, Computer-aided designing, supporting decision making to develop communication channels, robotics, space ships, ... etc.

\section{DETERMINATION OF GEODESIC BY THE EULER-LAGRANGE SYSTEM}

Let $\mathrm{z}=\mathrm{f}(\mathrm{x}, \mathrm{y})$ be a surface in space. The general form of the standard of this surface:

$$
\begin{aligned}
& \mathrm{g}=\mathrm{Edx}^{2}+2 \mathrm{Fdxdy}+\mathrm{Gdy}^{2} \\
& \text { where } \\
& \mathrm{G}=1+\mathrm{f}_{\mathrm{y}}^{2}, \mathrm{E}=1+\mathrm{f}_{\mathrm{x}}^{2}, \mathrm{~F}=\mathrm{f}_{\mathrm{x}} \mathrm{f}_{\mathrm{y}} \\
& \mathrm{f}_{\mathrm{x}}=\frac{\partial}{\partial x}, \mathrm{f}_{\mathrm{y}}=\frac{\partial}{\partial y}
\end{aligned}
$$

The determination of the geodesics of a metric $\mathrm{g}(\mathrm{x}, \mathrm{y})$ can be done by the integration of the Euler-Lagrange system

$$
\begin{aligned}
& d 2 x / d t^{2}+\Gamma_{11}^{1}(d x / d t)^{2}+2 \Gamma_{12}^{1}(d x / d t)(d y / d t)+\Gamma_{22}^{1}+(d y / d t)^{2} \\
& d 2 y / d t^{2}+\Gamma_{11}^{2}(d x / d t)^{2}+2 \Gamma_{12}^{2}(d x / d t)(d y / d t)+\Gamma_{22}^{2}+(d y / d t)^{2}
\end{aligned}
$$

The Christoffel symbols are given by:

$$
\begin{aligned}
& \Gamma_{i j}^{1}(x, y)=\frac{1}{2}\left(g^{11}\left(\frac{\partial g_{1 j}}{\partial x_{1}}+\frac{\partial g_{11}}{\partial x_{j}}-\frac{\partial g_{i j}}{\partial x_{1}}\right)+\right. \\
& g^{21}\left(\frac{\partial g_{2 j}}{\partial x_{1}}+\frac{\partial g_{12}}{\partial x_{j}}-\frac{\partial g_{i j}}{\partial x_{2}}\right) \\
& \Gamma_{i j}^{2}(x, y)=\frac{1}{2}\left(g^{12}\left(\frac{\partial g_{1 j}}{\partial x_{1}}+\frac{\partial g_{11}}{\partial x_{j}}-\frac{\partial g_{i j}}{\partial x_{1}}\right)+\right. \\
& g^{22}\left(\frac{\partial g_{2 j}}{\partial x_{1}}+\frac{\partial g_{12}}{\partial x_{j}}-\frac{\partial g_{i j}}{\partial x_{2}}\right)
\end{aligned}
$$

for $\mathrm{i}, \mathrm{j}=1.2$. 
The $\mathrm{g}_{\mathrm{ij}}$ are the elements of the matrix of the coefficients of the metric, that is to say:

$$
\begin{aligned}
& g=d s^{2}=g_{11} d x^{2}+2 g_{12} d x d y+g_{22} d y^{2} \\
& g_{i j}=\left[\begin{array}{ll}
g_{11} & g_{12} \\
g_{21} & g_{22}
\end{array}\right]=\left[\begin{array}{ll}
E & F \\
F & G
\end{array}\right]
\end{aligned}
$$

The gij are the elements of the inverse matrix of (gij).

Each solution of Euler-Lagrange system is a geodesic, and it is determined by a starting point $(\mathrm{x} 0, \mathrm{y} 0)$ and an initial velocity $(\mathrm{dx} / \mathrm{dt}, \mathrm{dy} / \mathrm{dt}) \mathrm{t}=0$ at that point.

Examples

- For the Euclidean metric $\mathrm{g}=\mathrm{dx} 2+\mathrm{dy} 2$

We have

$$
\left(g_{i j}\right)=\left[\begin{array}{ll}
g_{11} & g_{12} \\
g_{21} & g_{22}
\end{array}\right]=\left[\begin{array}{ll}
1 & 0 \\
0 & 1
\end{array}\right]
$$

$$
\text { Ilere } \mathrm{I}_{t j}^{k}=0 \text { for } i, j, k=1,2
$$

The Euler-Lagrange equations corresponding to this metric is:

$$
\left\{\begin{array}{l}
x^{\prime \prime}(t)=0 \\
y^{\prime \prime}(t)=0
\end{array}\right.
$$

The solutions are lines.

- Let $\mathrm{g}$ be the metric of Poincaré defined on the half plane y> 0 by

$$
g(x, y)=\frac{d x^{2}+d y^{2}}{y^{2}}
$$

The Euler-Lagrange equations are:

$$
\left\{\begin{array}{c}
x^{\prime \prime}(t)=\frac{2 x^{\prime}(t) y^{\prime}(t)}{y(t)} \\
y^{\prime \prime}(t)=\frac{y^{\prime 2}(t)-x^{\prime 2}(t)}{y(t)}
\end{array}\right.
$$

The solution systems are semi circles centered on the $\mathrm{x}$ axis and half-lines parallel to the y axis.

\section{THE NUMERICAL RESOLUTION OF THE EULER-LAGRANGE EQUATIONS}

In general, we are compelled to use a numerical method to integrate the Euler Lagrange system, which has a geodesic solution starting from a point $(\mathrm{x} 0, \mathrm{y} 0)$ with an initial velocity $\mathrm{v} 0$ at this point; to draw several geodesics starting from the same point. It suffices to vary the initial velocity, and to determine the solution which starts from one point and passes through another. To achieve this, it is necessary to find the suitable initial velocity which gives this solution.

Here is a regressive method of finding the initial velocity for an Euler-Lagrange solution passing through two points.

Assuming that we have two points $\mathrm{P}(\mathrm{xp}, \mathrm{yp})$ and $\mathrm{Q}$ (xq, yq) and $y p=y q=y$. The problem of connecting these two points for a given metric using a geodesic line can be solved by the integration of Euler-Lagrange system.

This problem requires finding the angle $(\theta)$ of the initial velocity beam at the point $\mathrm{P}: \mathrm{v} 0(\cos \theta, \sin \theta)$ in order for the solution to pass from point $\mathrm{P}$ to $\mathrm{Q}$.
Assuming that angle $\theta$ led to the point $\mathrm{P}_{1}\left(\mathrm{x}_{1}, \mathrm{y}^{\prime}\right)$, and the angle $\theta 2$ led to the point $\mathrm{P}_{2}\left(\mathrm{x}_{2}, \mathrm{y}^{\prime}\right)$ (we can presume that $\mathrm{x}_{\mathrm{q}}$ is located between $x_{1}$ and $\left.x_{2}\right)$. Let's symbolize that $\theta 1 \rightarrow x(\theta 1)$, and $\theta 2 \rightarrow \mathrm{x}(\theta 2)$.

When the distance between $x_{1}$ and $x_{2}$ is small enough, we can give the formula $\theta=0.5(\theta 1+\theta 2)$ to the angle $\theta$ that leads us to the point $(\mathrm{x}, \mathrm{y})$.

If $x>x(q)$, then we repeat with the angles $\theta 1$ and $\theta$, and if $x<$ $x(q)$, then we repeat with the angles $\theta$ and $\theta 2$. We repeat this procedure until $\mathrm{x}$ is close enough to $\mathrm{x}_{\mathrm{q}}$.

This method for determining the initial velocity angle converges to the point $\mathrm{xq}$ because $\mathrm{x} 1$ and $\mathrm{x} 2$ are located in two different directions (Figure 1).

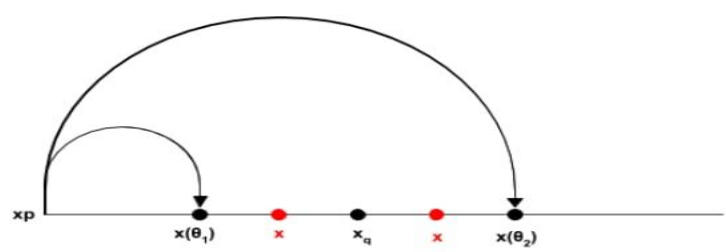

Figure 1. Finding the angle $(\theta)$ of the initial velocity

We summarize this by the following steps

if $(\operatorname{abs}(x 1-x q)>0.5)$ and $(a b s(x q-x 2)>0.5)$ then

begin

teta $=($ teta $1+$ teta 2$) / 2$;

Runge_kutta(teta,x); // procedure uses Runge Kutta algorithm

if $x>x q$ then sol(teta1, teta, $x 1, x)$

else if $x<x q$ then sol(teta,teta2, $x, x 2)$; end

An integrated computer program was developed to draw the geodesic curve of many metrics, and the following result were obtained

- Fast generation of results when the metrics are not sensitive to the initial speed changes applied to the first point, but solutions are slow or impossible in the opposite cases.

\section{NUMERICAL METHOD TO DETERMINE THE SHORTEST PATH}

I want to introduce a numerical method based on random modifications to determine the shortest path.

Two points $\mathrm{P} 0$ and $\mathrm{Pn}$ can be taken at the computer screen and a random set of points $\{\mathrm{Pi}\}$, for $\mathrm{i}=1,2, . ., \mathrm{n}-1$ can be taken between them to perform random transitions of each of these points in random directions, in a square named "square of the displacement". The total length of the curve is then tested with each transition according to the given metric, and the displacement is adopted if this transition reduces the total length of the curve.

The calculation of the length of the separate curve will be approximate for the length of the true curve when the number of points supported $\{\mathrm{Pi}\}$ is large enough [3, 4].

Here are the practical steps for the proposed method

1. Input $\mathrm{P} 0, \mathrm{Pn}$, the metric of surface, and length the square of displacement

2. plot a random discrete curve between P0 and Pn

3. for each point $\mathrm{Pi} ; \mathrm{i}=1$ to $\mathrm{n}$ 
begin

3.1. calculate the curved length (P0 to $\mathrm{Pn})$

3.2. move the point $\mathrm{Pi}$ in random direction, and inside the square of displacement

3.3. the position is adopted if the length of the curve is reduced

end

repeat steps 3 until the length settles.

The random movement of points will be done by using the random number generator of the computer. The point $\mathrm{P}(\mathrm{x}[\mathrm{i}]$, $y[i])$ can be randomly moved into a square centered at the point $\mathrm{P}$ and has the side $\mathrm{L}$ of choice, as follows:

$$
x[i]+=2 * L *(\text { random }-0.5) \text {; }
$$

$$
y[i]+=2 * L *(\text { random }-0.5)
$$

This procedure makes it possible to generate random positions of the point $\mathrm{Pi}$ in a square of length $\mathrm{L}$ and centered at Pi.

\section{IMPLEMENTATION}

Here are some elements of the $\mathrm{C}++$ program illustrating the most important aspects of the method presented.

\section{- determination of the metric}

float Metric(float $x$, float $y$, float dx,float dy)

\{ return $(\mathrm{dx} * \mathrm{dx}+\mathrm{dy} * \mathrm{dy}) /\left(\mathrm{y}^{*} \mathrm{y}\right) ; / /$ Poincaré metric $\}$

- Calculation of lengths

To calculate the instantaneous length of the created discrete random curve, we apply the metric on each of the curve's points.

float L(float x1, float y1, float x2, float y2)

\{float dx, dy;

$\mathrm{dx}=\mathrm{x} 2-\mathrm{x} 1 ; \mathrm{dy}=\mathrm{y} 2-\mathrm{y} 1 ;$

return Metric (x1,y1,dx,dy);

\section{CONCLUSIONS AND FUTURE WORK}

The need for developing novel methods for finding the shortest paths between two points on a surface is evident and necessary. In the presented research, the limitations of conventional methods of approaching this problem were tested and presented. A numerical method based on random changes was then introduced to overcome the limitations of the current methods.

By tackling some examples with known solutions, we obtained separate curves very close to the shape and length of the real curves, and the presented method has proved to be feasible and reliable in generating robust solutions, especially when the number of points forming the curve is big enough.

The issue of finding the shortest path between two points of a surface is beneficial and significant in various fields and applications. For instance, the presented study is applicable and valuable to several applications, such as mobile networks, Computer-aided designing, and robotic. More work is being pursued to further develop this method and apply it in various domains.

\section{REFERENCES}

[1] J.M. Beck, R.T. Farouki et J. k. Hinds. Surface analysis methods, I.E.E.E., pp 18-36, 1986. https://doi.org/10.1109/MCG.1986.276587

[2] M.P. Do Carmo. Differential geometry of curves surfaces, Prentice Hall, Englewood Cliffs, N. J., 1976

[3] Ahmad Tayyar, Mohammad Ali Eljinini. Ontology-based generation of random paths Between two points, (JATIT). E-ISSN: 1817-3195. Vol. 96, 2018

[4] Ahmad Tayyar, Mohammad Ali H. Eljinini, Ontology-Based Generation of Random Paths between Two Points, Journal of Theoretical and Applied Information Technology (JATIT). E-ISSN: 1817-3195. Vol. 96, 2018

[5] Ahmad. Tayyar, "Generating Random Paths between Two Points in Space: Proposed Algorithm", Proceedings of the International Conference on Computer Science, Computer Engineering, and Social Media, Thessaloniki, Greece, 2014.

[6] A. Zakaria , M. S. N. Ibrahim, Numerical Performance Evaluation of Savonius Rotors by Flow-driven and Sliding-mesh Approaches, International Journal of Advanced Trends in Computer Science and Engineering, Volume 8, N0. 1, January-February 2019 https://doi.org/10.30534/ijatcse/2019/10812019

[7] D. Hema Latha , Prof. P. Premchand, Estimating Software Reliability Using Ant Colony Optimization Technique with Salesman Problem for Software Process, International Journal of Advanced Trends in Computer Science and Engineering, Volume 7, N0. 2, March April 2018 https://doi.org/10.30534/ijatcse/2018/04722018 